\title{
RecQ5 Interacts with Rad51 and Is Involved in Resistance of Drosophila to Cisplatin Treatment
}

\author{
Sayako Maruyama, ${ }^{a}$ Noriko Ohkita, ${ }^{b}$ Minoru Nakayama, ${ }^{a, b ; \dagger}$ Eiko Akaboshi ${ }^{c}$ Takehiko Shibata,${ }^{a}$ \\ Eishi Funakoshi, ${ }^{b, d}$ Kenji Takeuchi, ${ }^{b}$ Fumiaki Ito, ${ }^{b}$ and Katsumi Kawasaki*,a,b,d \\ ${ }^{a}$ Cellular and Molecular Biology Laboratory, RIKEN; 2-1 Hirosawa, Wako, Saitama 351-0198, Japan: ${ }^{b}$ Division of \\ Biochemistry, Graduate School of Pharmaceutical Sciences, Setsunan University; 45-1 Nagaotoge, Hirakata, Osaka \\ 573-0101, Japan: ${ }^{c}$ Laboratories for Integrated Biology, Graduate School of Frontier Biosciences, Osaka University; \\ 1-3 Yamadaoka, Suita, Osaka 565-0871, Japan: and ${ }^{d}$ Division of Molecular Biology, Department of Life Science, \\ Faculty of Science and Engineering, Setsunan University; 17-8 Ikedanaka, Neyagawa, Osaka 572-8508, Japan. \\ Received June 21, 2012; accepted August 28, 2012
}

RecQ5 is a member of the RecQ family of DNA helicases. There are 5 RecQ members in humans. Defects in 3 of them, i.e., BLM, WRN, and RTS, cause Bloom, Werner, and Rothmund-Thomson syndromes, respectively. RECQL1 and RECQL5 have not been associated with any human disease, and their precise roles are unknown. Our previous study suggests that the lack of RecQ5, which is the Drosophila homolog of RECQL5, leads to the accumulation of DNA double-stranded breaks (DSBs). It is possible that RecQ5 is involved in DSB repair. However, little is known about this possible function of RecQ5 in DSB repair. Here, we report that Rad51 protein, which plays a critical role in DSB repair, interacted with RecQ5 in vitro and in vivo in Drosophila. The Rad51 protein interacted with the $\mathrm{C}$-terminal region of RecQ5, as shown by the yeast two-hybrid method. Moreover, the $\mathrm{C}$-terminal region of the RecQ5 protein and the central region of Rad51 interacted directly and specifically when examined by the glutathione- $S$-transferase pull-down method. Consistent with these results, when RecQ5 and Rad51 were co-expressed in Drosophila cells in culture, they became co-localized in nuclei and could be co-immunoprecipitated. Furthermore, RecQ5-deficient flies (recq5) were more sensitive to the chemotherapeutic agent cisplatin compared with wild-type ones. Also, Rad51 mutants ( rad51) were more sensitive to cisplatin, with sensitivity similar to that of recq5 rad51 double mutants. These data suggest that RecQ5 and Rad51 in Drosophila functioned for survival after the flies had been treated with cisplatin.

Key words RecQ; Rad51; cisplatin; repair; DNA damage; Drosophila

RecQ5 is a member of the RecQ family of DNA helicases, in which there are 5 members in humans. Defects in 3 of them, i.e., BLM, WRN, and RTS, cause Bloom, Werner, and Rothmund-Thomson syndromes, respectively, in humans. Genome instability and predisposition to cancer is a common feature of many RecQ helicase-deficient cells. Although RecQL1 and RecQL5 (human homolog of Drosophila RecQ5), the other 2 members of the RecQ family in humans, have not been associated with any human genetic diseases, Recql5knock out mice show susceptibility to cancer, ${ }^{1)}$ suggesting that RecQ5 may also play an important role in preventing cancer. However, the precise role of RecQ5 remains unknown.

In Drosophila, the frequencies of spontaneous and induced chromosomal aberrations are increased in RecQ5-mutant neuroblasts. ${ }^{2}$ These data imply that double-stranded break (DSB) damage to DNA accumulates spontaneously in RecQ5 mutants. $^{2)}$ Furthermore, the loss of maternally-derived RecQ5 leads to spontaneous mitotic defects in syncytial embryos. These mitotic defects are derived from anaphase DNA bridges, which link pairs of daughter nuclei. These nuclei concurrently exit from the cycle and are eliminated by Drosophila checkpoint kinase 2 tumor suppressor homolog (DmChk2)dependent centrosome inactivation. ${ }^{3)}$ DmChk2 responds to DSB DNA lesions. ${ }^{4)}$ These findings suggest that the lack

The authors declare no conflict of interest.

${ }^{\dagger}$ Present address: Department of Molecular Biosciences, Faculty of Life Sciences, Kyoto Sangyo University; Motoyama, Kamigamo, Kita-ku, Kyoto 603-8555, Japan. of RecQ5 results in DSBs, which are recognized by the Chk2-checkpoint. However, little is known about the role of RecQ5 in DSB repair.

Cisplatin, which is cis-diamminedichloroplatinum(II), is a chemotherapeutic drug widely used for the treatment of several solid tumors, such as non-small-cell lung, ovarian, and testicular cancers. The mechanistic pathway by which this chemical exerts its function involves the induction of covalently linked DNA adducts. ${ }^{5)}$ Among these adducts, intrastrand $\mathrm{G}-\mathrm{G}$ cross-links ( $65 \%$ of the adducts) are the major lesions induced by this drug, ${ }^{6,7)}$ and are considered to be responsible for the chemotherapeutic effects of cisplatin. ${ }^{78}$ It is believed that the major pathway involves a DSB intermediate that is repaired by homologous recombination. ${ }^{9)}$ The recombination-repair system is necessary for DSB repair following cross-link removal. ${ }^{10)}$ Rad51 plays critical roles in DSB repair $^{11)}$ and DNA interstrand cross-link repair in eukaryotes. $^{12)}$ Previously, we showed that cisplatin increased the level of RecQ5 protein in S2 cells. ${ }^{13)}$ It is thus possible that RecQ5 responds appropriately to DNA damage induced by cisplatin.

In this report, to address the role of RecQ5 in DNA repair, we analyzed the relationship between RecQ5 and Rad51, both in vitro and in vivo. Furthermore, we measured the sensitivity of recq5, rad51, and recq5 rad51 Drosophila mutants to cisplatin. The results suggest that, in Drosophila, RecQ5 cooperated with Rad51 and was involved in the resistance to DNA damage induced by cisplatin. 


\section{MATERIALS AND METHODS}

Yeast Two-Hybrid Method To generate pASRECQ5 $\Delta N$, we cloned a DNA fragment corresponding to amino acids 464-1058 aa of RecQ5 (RecQ5 C, Fig. 1A) into pAS-2 (Clontech), as described earlier. ${ }^{14)}$ For pACT2-Rad51, Drosophila Rad51 cDNA was inserted into BamHI-SacI sites of pACT2 (Clontech). Yeast strain Y190 was transformed with pASRECQ5 $\triangle \mathrm{N}$ to express the Gal4 DNA binding domain (Gal4BD)-RECQ5 $\triangle \mathrm{N}$ fusion protein as described earlier. ${ }^{14)}$ The transformed clones were checked for growth on $\operatorname{Trp}^{-}$ $\mathrm{Leu}^{-} \mathrm{His}^{-}$medium containing $25 \mathrm{~mm} 3$-aminotriazole and assayed for $\beta$-galactosidase activity by performing the colony-lift assay.

Colony-Lift Filter Assay Fresh colonies were lifted onto Whatman \#5 filters. After the filters had been frozen completely in liquid nitrogen, they were incubated at $30^{\circ} \mathrm{C}$ in $100 \mathrm{~mm} \mathrm{NaPO}_{4}$ (pH 7.0) containing $10 \mathrm{~mm} \mathrm{KCl,} 1 \mathrm{~mm}$ $\mathrm{MgSO}_{4}, 0.3 \% \beta$-mercaptoethanol, and $0.3 \mathrm{mg} / \mathrm{mL}$ 5-bromo-4chloro-3-indolyl-D-galactopyranoside, as described earlier. ${ }^{14)}$

Construction of Rad51 Truncated Series Plasmid LD35801 (Berkeley Drosophila Genome Project), which contains the Sp6 promoter and Drosophila Rad51 cDNA cloned into the 5' EcoRI site and the 3' XhoI site of pOT2 (for Rad51-W, Fig. 2A), was digested with BanII and ligated to form Rad51-A (Fig. 2A). Plasmid LD35801 was digested with BstBI and $X c m \mathrm{I}$ and ligated to a linker (5'-CGGGCC ACA ACC AAA ATG GGC -3', 5'-CCC ATT TTG GTT GTG GCC -3') to produce Rad51-B (Fig. 2A). The Rad51-B was cut with $S p h$ I and FspI, blunted, and ligated to a linker to make Rad51-C (Fig. 2A). For Rad51-D, Rad51-B was cut with BglII, and ligated to produce Rad51-D (Fig. 2A).

In Vitro Transcription/Translation The plasmids of the Drosophila Rad51 truncated series were used to generate $\left[{ }^{35} \mathrm{~S}\right]$ methionine-labeled proteins in the TNT-coupled reticulocyte system (Promega), as described earlier. ${ }^{15)}$ The reaction mixtures were incubated at $30^{\circ} \mathrm{C}$ for $90 \mathrm{~min}$, and the labeled proteins were immediately used for the glutathione- $S$-transferase (GST) pull-down assay.

GST Pull-Down Assay The C-terminal region (464-1058 aa) of RecQ5 fused with GST protein (GST-RecQ5 C) was prepared as described earlier. ${ }^{14)}$ In vitro synthesized $\left[{ }^{35} \mathrm{~S}\right]$ methionine-labeled Rad51 protein was incubated with GST fusion protein $(1 \mu \mathrm{g})$ bound to glutathione Sepharose 4B beads (10 $\mu \mathrm{L}$, Amersham Biosciences) in $150 \mu \mathrm{L}$ of GST binding buffer $(50 \mathrm{~mm}$ potassium phosphate $\mathrm{pH} 7.5$, containing $150 \mathrm{~mm}$ $\mathrm{KCl}, 1 \mathrm{~mm} \mathrm{MgCl}_{2}, 10 \%$ glycerol, $1 \%$ Triton X-100, and protease inhibitors [Roche]) for $2 \mathrm{~h}$ at $4^{\circ} \mathrm{C}$. The beads were then washed 3 times in GST binding buffer, and the labeled proteins were separated by sodium dodecyl sulfate (SDS) $12 \%$ polyacrylamide gel electrophoresis. ${ }^{15)}$ The gels were dried and analyzed by using a BAS2500 Imaging plate reader (Fuji), as described earlier. ${ }^{14)}$

Immunocytology and Co-immunoprecipitation For construction of pCaSpeR-hs-3HA-RecQ5 (RecQ5 8-1058 aa), the ApaLI-AseI fragment of RecQ5 cDNA from LD21474 (Berkeley Drosophila Genome Project) was ligated into the StuI site of pCaSpeR-hs (a gift from Masayuki Miura). The resulting plasmid DNA was cut with HapI and SacII, and ligated to synthetic DNA (5'-CACAACCAA AAT GTA CCC CFACG ATG TGC CCG ATT ACG CCA TGT ACC GCTAC GAT GTG
CCC GAT TAC GCC ATG TAC CCC TAC GAC GTG CCC GAT TAC GCC GGC-3', 5'-CGG CGT AAT CGG GCA CGT CGT AGG GGT ACATG GCG TAA TCG GGC ACA TCG TAG CGG TACAT GGC GTA ATC GGG CAC ATC GTA GGG GTA CAT TTT GGT TGT G-3').

For construction of pCaSpeR-hs-FLAG-Rad51 (Rad51 11-336 aa), the EarI-AseI fragment of Rad51 $\mathrm{cDNA}^{16)}$ and synthetic DNA (5'-CACAACCAAAATGGA TTACAAGGA TGA CGA CGA TAA G-3', 5' 5TA GCT TAT CGT CGT CGT CAT CCT TAA TCC ATT TTGGTTGTGGC-3') were ligated to $S a c \mathrm{II}-X b a \mathrm{I}$ sites in pCaSpeR-hs.

S2 cells were transfected by use of a SuperFect transfection kit (QIAGEN) and incubated at $27^{\circ} \mathrm{C}$ for $1 \mathrm{~d}$. The heat-shock promoter was activated by $37^{\circ} \mathrm{C}$ treatment twice, for $30 \mathrm{~min}$ each time, with a $1-\mathrm{h}$ interval between treatments. Cells were harvested at $18 \mathrm{~h}$ after heat induction. These cells were stained with 1:1000-diluted anti-FLAG antibody M2 (Sigma), anti-HA antibody HA.11 (Santa Cruz), and $0.3 \mu \mathrm{g} / \mathrm{mL}$ Hoechst 33258. S2 cells (in 100-mm dishes) were collected and lysed in $600 \mu \mathrm{L}$ of lysis buffer $(50 \mathrm{~mm}$ Tris $-\mathrm{HCl}, 150 \mathrm{~mm} \mathrm{NaCl}$, $0.5 \%$ NP-40, $1 \mathrm{~mm}$ PMSF). After centrifugation, the pellet was extracted with $600 \mu \mathrm{L}$ of IP wash buffer (20mm Hepes [pH 7.4], $150 \mathrm{~mm} \mathrm{NaCl}, 1 \mathrm{~mm}$ EDTA, $1 \%$ polyoxyethylene (9) octylphenyl ether (NP-40), $1 \mathrm{~mm}$ phenylmethylsulfonyl fluoride (PMSF), protease inhibitor cocktail (complete mini Roche)) for $30 \mathrm{~min}$, and sheared by extrusion through a $25 \mathrm{G}$ needle. The supernatant $(180 \mu \mathrm{L})$ was precleaned with protein G Sepharose beads for $30 \mathrm{~min}$, mixed with $2 \mu \mathrm{L}$ of anti-HA antibody overnight, and then added to $20 \mu \mathrm{L}$ of protein $\mathrm{G}$ Sepharose. The precipitates were resuspended and washed 3 times with $400 \mu \mathrm{L}$ of IP wash buffer each time. The resulting pellet was resuspended in SDS sample buffer. Immunoblot analysis was carried out as described earlier. ${ }^{13)}$

Cisplatin Sensitivity Sensitivity of developing larvae to cisplatin (cis-diaminedichloroplatium(II), Sigma-Aldrich) was performed as described by Boyd et al. ${ }^{17)}$ Adults were crossed in plastic vials such that mutant and control classes were generated among the progeny. Adults were removed after $2 \mathrm{~d}$ of egg laying. After 1 additional day, $600 \mu \mathrm{L}$ of $0.05-0.50 \mathrm{~mm}$ cisplatin in water was added to the medium. To calculate sensitivity, we counted the adult progeny and then determined the ratio of mutant progeny to control progeny. Controls were balanced, heterozygous siblings in the same vial. The recq $5^{D l}$ $(\text { recq5 })^{2)}$ and $D f(3 L) f z-D 21$ (Drosophila Genetic Resource Center) are deficient in RecQ5. $D p(1 ; \mathrm{Y}) \mathrm{BS} ; r u^{l} s t^{l} e^{l} s p n-A^{l}$ $\mathrm{ca}^{l} / \mathrm{TM} 3, \mathrm{Sb}^{l}$ (rad51, Bloomington Drosophila Stock Center) contains a mutation for spindle-A, the Rad51 homolog, in Drosophila ${ }^{18)}$ For the double-mutant experiments, $D f(3 L)$ $f z-D 21-s p n-A^{1} / T M 6 B$ were crossed to recq $5^{D 1}-s p n-A^{1} / T M 3$. Progenies with the genotypes recq5 $5^{D 1}$-spn- $A^{1} / T M 6 B$ (control) and recq $5^{D 1}$-spn- $A^{1} / D f(3 L) f z-D 21-s p n-A^{1}$ (recq5 rad51 double mutant) were compared. Heterozygosity for recq5 did not affect the survival of rad51 mutants and vice versa (data not shown). At least 500 individuals were counted for each point. $D f(3 L) f z-D 21-s p n-A^{1} / T M 6 B$ and recq $5^{D 1}-s p n-A^{1} / T M 3$ flies were generated by recombination between $s p n-A^{1}$ and $D f(3 L) f z-D 21$ or recq $5^{D 1}$. $)$

\section{RESULTS}

Physical Interaction between Rad51 and RecQ5 
(A)

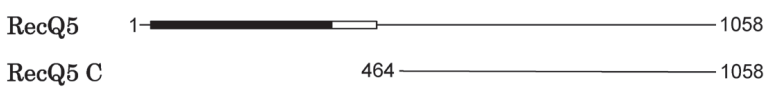

(B)

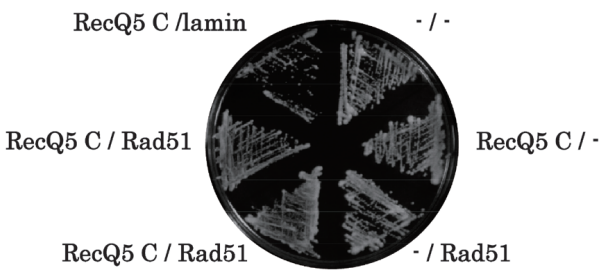

(C)

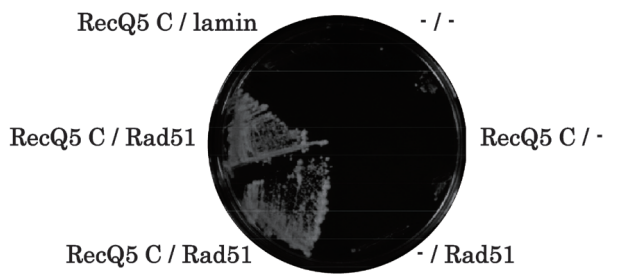

(D)

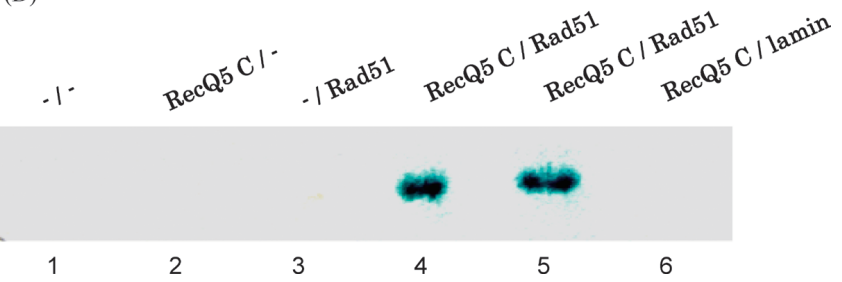

Fig. 1. Rad51 Interacts Specifically with RecQ5 in the Yeast Two-Hybrid System

(A) Schematic representation of Drosophila RecQ5 molecule and RecQ5 C-terminal region (RecQ5 $\mathrm{C}$ ). The closed and open boxes indicate the helicase domain and the extended region homologous in the RecQ family, respectively. (B, C) Y190 yeast cells containing lacZ and HIS3 reporter genes were co-transformed with plasmids pAS2-1 and pACT2 (-/-), pASRECQ5 $\triangle \mathrm{N}$ and pACT2 (RecQ5 C/-), pAS2-1 and pACT2-Rad51 (-/Rad51), pASRECQ5 $\Delta \mathrm{N}$ and pACT2-Rad51 (RecQ5 $\mathrm{C} / \operatorname{Rad} 51$, duplicate) or pASRECQ5 $\Delta \mathrm{N}$ and pLAM5'-1 (RecQ5 C/lamin). The transformed clones were incubated on $\mathrm{SD} /-\operatorname{Trp} /-$ Leu (B) or $\mathrm{SD} /-$ HIS (C) plates. The $\beta$-galactosidase activities were measured by performing the colony-lift filter assay (D) as described in Materials and Methods.

Previously we reported that Drosophila RecQ5 can complement several phenotypes of $s g s 1$ in yeast, including the synthetic growth defect with srs2, the suppression of slow growth in top3, the hypersensitivity to hydroxyurea and methyl methanesulfonate, and the elevated frequency of homologous recombination and sister chromatid exchange. ${ }^{19)}$ These past findings suggest that RecQ5 exhibits an evolutionally conserved RecQ function in vivo. The above phenotypes of the $s g s 1$ mutation is related to Rad51-dependent homologous recombination..$^{20,21)}$ In addition, Sgs1, the only RecQ of Saccharomyces cerevisiae, interacts with yeast $\operatorname{Rad} 51 .{ }^{22}$ It has thus been speculated that RecQ5 interacts with yeast Rad51, which is $77 \%$ similar to Drosophila Rad51. ${ }^{16)}$ Therefore, we examined whether Drosophila Rad51 protein could interact with the Drosophila RecQ5 by using the yeast two-hybrid method. Drosophila RecQ5 consists of a short N-terminal region, a helicase domain, and a unique $\mathrm{C}$-terminal region ${ }^{15}$ (Fig. 1A). The helicase domain in RecQ5 is in common with
(A)

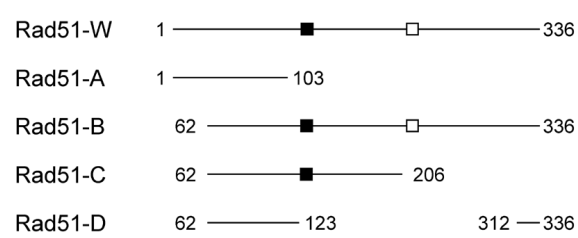

(B)

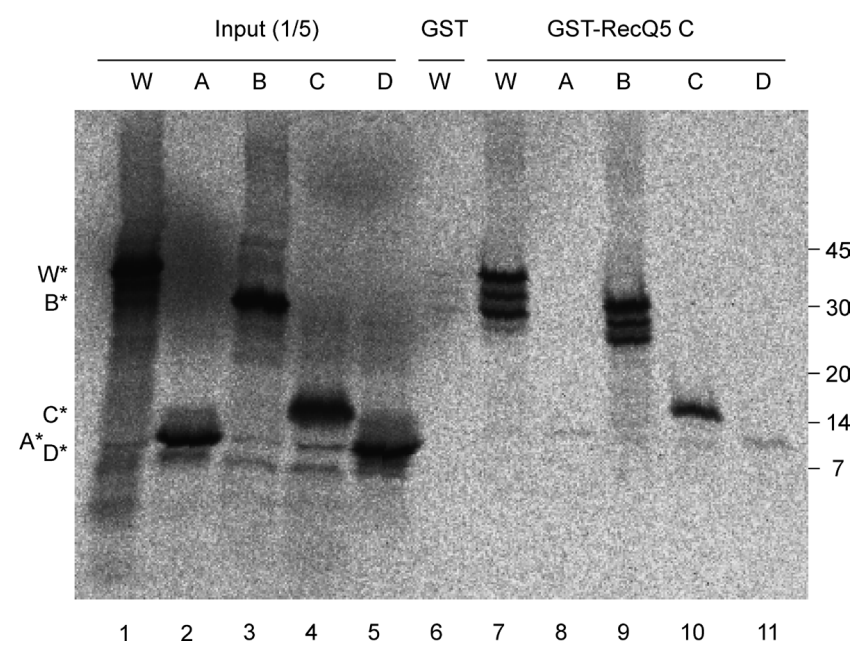

Fig. 2. GST-RecQ5 C Pull-Down of Rad51

(A) Schematic representation of truncated Drosophila Rad51 molecules. Closed and open boxes indicate Walker motifs A and B, respectively. ${ }^{37)}$ (B) Physical interaction between Rad51 protein and RecQ5. A series of $\left[{ }^{35} \mathrm{~S}\right]$-methionine-labeled Rad51 proteins were pulled down by GST-fused RecQ5 protein. Lanes 1, 6, and 7, whole Rad51-W (1-336 aa); lanes 2 and 8, Rad51-A (1-103 aa); lanes 3 and 9, Rad51-B (62-336 aa); lanes 4 and 10, Rad51-C (62-206 aa); lanes 5 and 11, Rad51-D (62-123, 312-336 aa); lanes $1-5,20 \%$ of input; lane 6 , pull-down by GST alone; lanes 7-11, pull-down by GST-RecQ5 C. Bars at the right side of the panel indicate molecular weight markers $(\mathrm{kDa})$. The asterisks at the left side of the panel correspond to the expected molecular weights of the truncated Rad51 proteins ( ${ }^{*}$, $\left.A^{*}, B^{*}, C^{*}, D^{*}\right)$.

that in the RecQL1, BLM, WRN, and RTS proteins, ${ }^{15)}$ and the relatively large $\mathrm{C}$-terminal region is likely important in functionally distinguishing these members of the RecQ helicase family. To analyze the role of the C-terminal region in RecQ5, we previously performed a yeast two-hybrid method in a Drosophila embryo cDNA library by using the C-terminal region (residues 464-1058 aa) of RecQ5 as bait. ${ }^{14)}$ Expression of the C-terminal region of RecQ5 as a Gal4BD fusion protein (Gal4BD-RecQ5 C) was confirmed by immunoblot analysis of yeast cell lysates with anti-RecQ5 and anti-Gal4BD antibodies (data not shown). We used Gal4BD-RecQ5 C for simultaneous co-transformation with Gal4AD-Rad51 plasmid, empty vector or pAS-lamin as a negative control in the Y190 strain (Fig. 1B). Together with Gal4BD-RecQ5 C, the Gal4AD-Rad51 plasmid activated the HIS3 reporter gene (Fig. $1 C$, RecQ5 C/Rad51), and also the LacZ reporter gene (Fig. 1D, RecQ5 C/Rad51). Neither empty vector nor pAS-lamin activated them with Gal4BD-RecQ5 C (Figs. 1C,D, RecQ5 C/- \& RecQ5 C/lamin). Gal4AD-Rad51 did not activate the reporter genes without RecQ5 C (Figs. 1C,D, -/Rad51). Thus, Gal4AD-Rad51 and Gal4BD-RecQ5 C specifically activated both the HIS3 reporter gene and lacZ reporter gene in the yeast two-hybrid system, suggesting specific interaction 


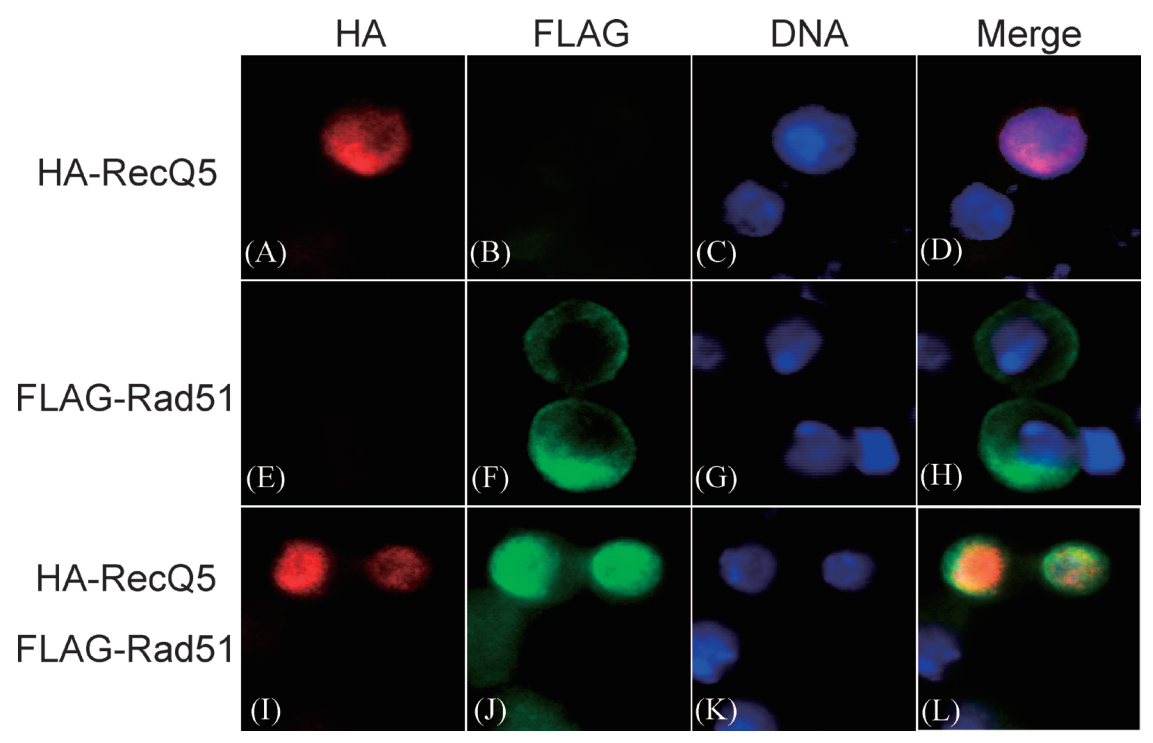

Fig. 3. Immuno Co-localization of RecQ5 and Rad51 to the Nuclei

Anti-HA staining of a field of cells transfected with HA-tagged RecQ5 (A), FLAG-tagged Rad51 (E) and both (I). The same field stained with anti-FLAG antibody $(\mathrm{B}, \mathrm{F}, \mathrm{J})$ and with Hoechst 33258 for DNA $(\mathrm{C}, \mathrm{G}, \mathrm{K})$. The merged image is also shown (D, H, L). Two cells can be seen expressing FLAG-Rad51 and HA-RecQ5 (I,J,L). In both cells the proteins had been localized to the nucleus.

between Rad51 and the C-terminal region of RecQ5 (Fig. 1).

RecQ5 Binding Domain in Rad51 To confirm the physical interaction of Rad51 with RecQ5, we performed a GST pull-down assay by using various fragments of Rad51 (Fig. 2A). The whole region of the Rad51 protein bound to GST-fused to the C-terminal region of the RecQ5 protein (Fig. 2B, lane 7), whereas GST alone did not pull down the whole Rad51 (Fig. 2B, lane 6), suggesting specific interaction between $\mathrm{Rad} 51$ and the $\mathrm{C}$-terminal region of RecQ5. The C-terminal region of RecQ5 also bound to the central domain, 62-336, 62-206 aa of Rad51 (Fig. 2B, lanes 9, 10, respectively), but not to the N-terminal (1-103, 62-123 aa) and C-terminal (312-336 aa) regions of Rad51 (Fig. 2B, lanes 8, 11), in vitro. This pattern indicates that amino acid residues 124-206 of Rad51 were important for the interaction with RecQ5.

RecQ5 Is Localized in Nuclei Next, we checked the localization of RecQ5 and Rad51 in Drosophila cells in culture. S2 cells were transfected with HA-tagged RecQ5 (HA-RacQ5 [8-1058 aa], Figs. 3A-D), FLAG-tagged Rad51 (FLAG-Rad51 [11-336 aa]), Figs. 3E-H) or both (Figs. 3I-L), and then stained with anti-HA antibody, anti-FLAG antibody, and Hoechst33258. RecQ5 was detected in the nucleoplasm (Fig. 3A). ${ }^{23-25)}$ Rad51 itself has no detectable nuclear localization signal (NLS), ${ }^{26)}$ and so was not found in the nucleus; however, significant levels of it were found in the cytoplasm (Fig. 3F), as similarly reported previously. ${ }^{26,27)}$ HA-RecQ5 and FLAG-Rad51 were co-localized in the nuclei (Figs. 3I-L). These data suggest that Rad51 became localized together with RecQ5 in the S2 nuclei.

HA-RecQ5 Co-immunoprecipitates with FLAG-Rad51 To test whether Rad51 would bind to RecQ5 in vivo, we prepared extracts of S2 cells that had been transfected with FLAG-Rad51, HA-RecQ5 or both. The proteins were immunoprecipitated with anti-HA antibody and then subjected to immunoblotting using anti-HA and anti-FLAG antibodies. The anti-HA antibody immunoprecipitated HA-RecQ5 (Fig. 4, lane 8), whereas it did not pull down FLAG-Rad51 when no

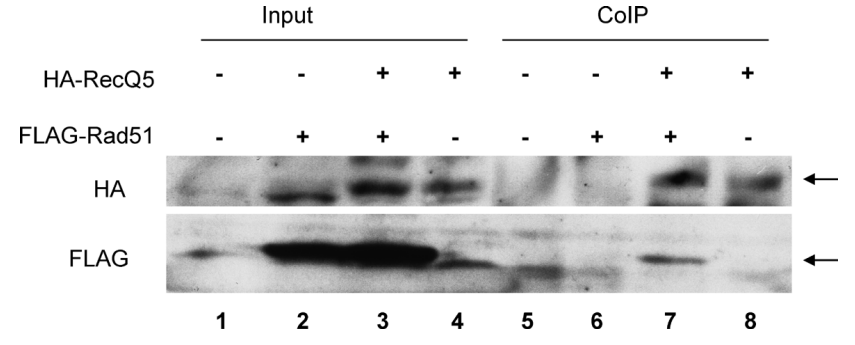

Fig. 4. HA-RecQ5 Co-immunoprecipitates FLAG-Rad51

S2 cells were transfected with FLAG-tagged Rad51 (lanes 2, 6), HA-tagged RecQ5 (lanes 4, 8) or both (lanes 3, 7). Cell extracts were prepared, immunoprecipitated with anti-HA antibody and analyzed by Western blotting using anti-HA antibody (HA) or anti-FLAG (FLAG), as described in Materials and Methods.

HA-RecQ5 was used for co-transfection (Fig. 4, lane 6). When cells were co-transfected with FLAG-Rad51 and HA-RecQ5, anti-HA antibody immunoprecipitated HA-RecQ5 along with FLAG-Rad51 (Fig. 4, lane 7). These data suggest that RecQ5 interacted physically with Rad51 in S2 cells.

Cisplatin Sensitivity of recq5, rad51, and recq5 rad51 Mutants Cisplatin is a chemotherapeutic agent that forms many different adducts with DNA, removal of some of which would require formation of a DNA DSB. ${ }^{28}$ ) Previously, we showed that cisplatin increases the level of RecQ5 protein in S2 cells. ${ }^{13)}$ To check the functional interaction between RecQ5 and Rad51, we measured the sensitivity of flies to cisplatin. Crosses were designed to produce heterozygotes and homozygotes. Cisplatin was added to the food of 2 nd larvae. After eclosion, the number of adult flies was counted and the observed number of homozygotes was compared with the expected number of homozygotes from the observed number of heterozygotes. As a result, the recq5 mutant was shown to be sensitive to cisplatin (Fig. 5), suggesting that RecQ5 was involved in resistance to DNA damage induced by cisplatin. The rad51 flies (mutation in Rad51) were even more sensitive to the drug, as were the double mutant, recq5 rad51, ones (Fig. 5). The addition of the RecQ5 mutation to the Rad51 mutation 


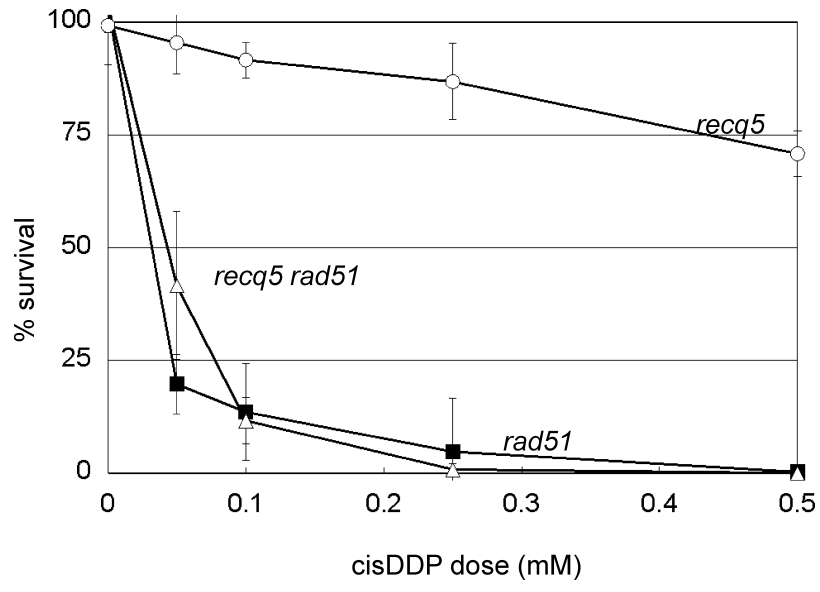

Fig. 5. Cisplatin Sensitivity of recq5, rad51, and recq5 rad51 Mutants

Survival of recq5 (open circle), rad51 (closed box), and recq5 rad51 (open triangle) mutants after treatment with cisplatin. Percent survival is given relative to the survival of wild-type controls (heterozygous siblings; see Experimental Procedures for details). Bars indicate standard deviation of at least 2 independent experiments Cisplatin was added to the medium as described in Materials and Methods.

did not appear to have any additive or synergistic effect on cisplatin sensitivity. These data suggest that RecQ5 and Rad51 in Drosophila functioned for survival after the flies had been treated with cisplatin.

\section{DISCUSSION}

Here, we have shown both in vitro and in vivo the interaction between Rad51 and RecQ5. The physical interaction between RecQ5 and Rad51 was confirmed by using 3 independent experimental methods, i.e., the yeast two-hybrid method (Fig. 1), GST pull-down method (Fig. 2), and co-immunoprecipitation (Fig. 4)

In Drosophila S2 cells in culture, Rad51 co-localized with RecQ5 in the nucleus (Fig. 3). Rad51 itself has no detectable NLS. ${ }^{26}$ A potential NLS with the sequence KRPRSQQENPESQPQKRPR exists in the C-terminus of human RecQ5 $\beta .^{24)}$ Nuclear entry of the Rad51 protein likely requires its interaction with another protein that contains a functional NLS, such as Brca2, ${ }^{27)}$ Rad51C, ${ }^{26)}$ or RecQ5.

RecQ5-deficient flies (recq5) were more sensitive to the chemotherapeutic agent cisplatin than the wild-type ones; and the Rad51 mutants (rad51) were more sensitive to cisplatin than the RecQ5 mutants. It is possible that the RecQ5 function is partially substituted by other proteins in affording resistance to cisplatin. Alternatively, Rad51 and RecQ5 may function in separate pathways for resistance to cisplatin. To distinguish between these possibilities, we examined cisplatin sensitivity in the recq5 rad51 double mutants. If these genes were required in separate pathways, the double mutants, which would be defective in both pathways, should show an additive or synergistic effect on cisplatin sensitivity. However, we found that these recq5 $\mathrm{rad} 51$ double mutants were almost identical in sensitivity to the rad51 single mutants (Fig. 5). These data imply a model in which RecQ5 would be required for a subset of Rad51-dependent repair of DNA damage caused by cisplatin, although other models could also account for it. Since cisplatin increases the level of RecQ5 protein in S2 cells, ${ }^{13)}$ Rad51 would cooperate with RecQ5 in response to
DNA damages in Drosophila.

Members of the RecQ family share homology in their RecQ helicase domain. Although the C-terminal regions of RecQ5, Sgs1, and BLM share little sequence homology, the C-terminal region of yeast Sgs1 (1299-1447 aa) interacts with yeast Rad51 in the yeast two-hybrid system. ${ }^{22)}$ The C-terminal region of human BLM (1267-1417 aa) also interacts with human Rad51 in the yeast two-hybrid system. ${ }^{22)}$ The C-terminal region of RecQ would be important for the interaction of Rad51 in the same species. The C-terminal region of HsRecQ5 binds with several proteins, such as HsRad51, ${ }^{29-32)}$ PCNA, ${ }^{29,31-33)}$ and RNPII. $^{32,34-36)}$ The C-terminal region of RecQ5 would thus be expected to coordinate RecQ5 function with that of other proteins in vivo.

Rad51-W (1-336 aa), Rad51-B (62-336 aa), and Rad51-C (62-206 aa) bound to GST-RecQ5 (464-1058 aa), whereas Rad51-A (1-103 aa) and Rad51-D (62-123, 312-336 aa) did not (Fig. 2B). These data suggest that the central region of Rad51 (124-206 aa) was important for the interaction of Rad51 with RecQ5. This region contains the walker A motif (Fig. 2A), ${ }^{37)}$ which corresponds to the ATP-binding site, and is conserved well in Rad51. Recently, a BRC repeat variant, named BRCv, was identified in HsRecQ5, and was shown to be essential for RecQ5 to interact with $\operatorname{Rad51.}{ }^{38)}$ BRC repeats interact with Rad51 and promote homologous recombination by regulating the Rad51 recombinase activity in the tumor suppressor protein Brca2 (breast cancer type 2 susceptibility protein). ${ }^{39)}$ Exceptionally, only Drosophila RecQ5 contains 2 putative BRCv repeats; whereas $\mathrm{BRCV}$ is present as a single copy in RecQ5 from most species. It is possible that tandem $\mathrm{BRCV}$ repeats in Drosophila RecQ5 would work synergistically to activate homologous recombination in nuclei as seen in Brca2. ${ }^{40)}$

Acknowledgments We gratefully acknowledge the Bloomington Drosophila Stock Center and Drosophila Genetic Resource Center in Kyoto Institute of Technology for providing the fly stocks used. This work was supported by the Science Research Promotion Fund from the Promotion and Mutual Aid Corporation for Private Schools of Japan.

\section{REFERENCES}

1) Hu Y, Raynard S, Sehorn MG, Lu X, Bussen W, Zheng L, Stark JM, Barnes EL, Chi P, Janscak P, Jasin M, Vogel H, Sung P, Luo G. RECQL5/Recq15 helicase regulates homologous recombination and suppresses tumor formation via disruption of Rad51 presynaptic filaments. Genes Dev., 21, 3073-3084 (2007).

2) Nakayama M, Yamaguchi S, Sagisu Y, Sakurai H, Ito F, Kawasaki $\mathrm{K}$. Loss of RecQ5 leads to spontaneous mitotic defects and chromosomal aberrations in Drosophila melanogaster. DNA Repair, 8 , 232-241 (2009).

3) Sakurai H, Okado M, Ito F, Kawasaki K. Anaphase DNA bridges induced by lack of RecQ5 in Drosophila syncytial embryos. FEBS Lett., 585, 1923-1928 (2011).

4) Takada S, Kelkar A, Theurkauf WE. Drosophila checkpoint kinase 2 couples centrosome function and spindle assembly to genomic integrity. Cell, 113, 87-99 (2003).

5) Friedberg EC, Walker GC, Siede W, Wood RD, Schultz RA, Ellenberger T. DNA Repair and Mutagenesis, ASM Press, Washington, D.C. (2006).

6) Eastman A. The formation, isolation and characterization of DNA adducts produced by anticancer platinum complexes. Pharmacol. 
Ther., 34, 155-166 (1987).

7) Kartalou M, Essigmann JM. Recognition of cisplatin adducts by cellular proteins. Mutat. Res., 478, 1-21 (2001).

8) Almeida GM, Duarte TL, Steward WP, Jones GD. Detection of oxaliplatin-induced DNA crosslinks in vitro and in cancer patients using the alkaline comet assay. DNA Repair (Amst.), 5, 219-225 (2006).

9) McHugh PJ, Spanswick VJ, Hartley JA. Repair of DNA interstrand crosslinks: molecular mechanisms and clinical relevance. Lancet Oncol., 2, 483-490 (2001).

10) Jachymczyk WJ, von Borstel RC, Mowat MR, Hastings PJ. Repair of interstrand cross-links in DNA of Saccharomyces cerevisiae requires two systems for DNA repair: the RAD3 system and the RAD51 system. Mol. Gen. Genet., 182, 196-205 (1981).

11) Shinohara A, Ogawa H, Ogawa T. Rad51 protein involved in repair and recombination in S. cerevisiae is a RecA-like protein. Cell, 69, 457-470 (1992).

12) Long DT, Räschle M, Joukov V, Walter JC. Mechanism of RAD51-dependent DNA interstrand cross-link repair. Science, $\mathbf{3 3 3}$, 84-87 (2011).

13) Nakayama M, Maruyama $S$, Kanda $H$, Ohkita N, Nakano K, Ito F, Kawasaki K. Relationships of Drosophila melanogaster RECQ5/ $\mathrm{QE}$ to cell-cycle progression and DNA damage. FEBS Lett., 580, 6938-6942 (2006).

14) Nakayama M, Quang NM, Matsumoto K, Shibata T, Ito F, Kawasaki K. RECQ5/QE helicase interacts with retrotransposon mdg3 gag, an HIV nucleocapsid-related protein. J. Health Sci., 52, 24-29 (2006).

15) Jeong SM, Kawasaki K, Juni N, Shibata T. Identification of Drosophila melanogaster RECQE as a member of a new family of RecQ homologues that is preferentially expressed in early embryos. Mol. Gen. Genet., 263, 183-193 (2000).

16) Akaboshi E, Inoue $\mathrm{Y}$, Ryo H. Cloning of the cDNA and genomic DNA that correspond to the recA-like gene of Drosophila melanogaster. Jpn. J. Genet., 69, 663-670 (1994).

17) Boyd JB, Golino MD, Nguyen TD, Green MM. Isolation and characterization of X-linked mutants of Drosophila melanogaster which are sensitive to mutagens. Genetics, 84, 485-506 (1976).

18) Staeva-Vieira E, Yoo S, Lehmann R. An essential role of DmRad51/ SpnA in DNA repair and meiotic checkpoint control. EMBO J., 22, 5863-5874 (2003).

19) Nakayama M, Kawasaki K, Matsumoto K, Shibata T. The possible roles of the DNA helicase and C-terminal domains in RECQ5/QE: complementation study in yeast. DNA Repair (Amst.), 3, 369-378 (2004).

20) Gangloff S, Soustelle C, Fabre F. Homologous recombination is responsible for cell death in the absence of the Sgs1 and Srs2 helicases. Nat. Genet., 25, 192-194 (2000).

21) Shor E, Gangloff S, Wagner M, Weinstein J, Price G, Rothstein R. Mutations in homologous recombination genes rescue top3 slow growth in Saccharomyces cerevisiae. Genetics, 162, 647-662 (2002).

22) Wu L, Davies SL, Levitt NC, Hickson ID. Potential role for the BLM helicase in recombinational repair via a conserved interaction with RAD51. J. Biol. Chem., 276, 19375-19381 (2001).

23) Sekelsky JJ, Brodsky MH, Rubin GM, Hawley RS. Drosophila and human RecQ5 exist in different isoforms generated by alternative splicing. Nucleic Acids Res., 27, 3762-3769 (1999).

24) Shimamoto A, Nishikawa K, Kitao S, Furuichi $\mathrm{Y}$. Human RecQ5 $\beta$, a large isomer of RecQ5 DNA helicase, localizes in the nucleoplasm and interacts with topoisomerases $3 \alpha$ and $3 \beta$. Nucleic Acids Res., 28, 1647-1655 (2000).

25) Popuri V, Ramamoorthy M, Tadokoro T, Singh DK, Karmakar
P, Croteau DL, Bohr VA. Recruitment and retention dynamics of RECQL5 at DNA double strand break sites. DNA Repair (Amst.), 11, 624-635 (2012).

26) Gildemeister OS, Sage JM, Knight KL. Cellular redistribution of Rad51 in response to DNA damage: novel role for Rad51C. J. Biol. Chem., 284, 31945-31952 (2009).

27) Davies AA, Masson JY, McIlwraith MJ, Stasiak AZ, Stasiak A, Venkitaraman AR, West SC. Role of BRCA2 in control of the RAD51 recombination and DNA repair protein. Mol. Cell, 7, 273282 (2001).

28) Bhattacharyya A, Ear US, Koller BH, Weichselbaum RR, Bishop DK. The breast cancer susceptibility gene BRCA1 is required for subnuclear assembly of Rad51 and survival following treatment with the DNA cross-linking agent cisplatin. J. Biol. Chem., 275, 23899-23903 (2000).

29) Li M, Xu X, Liu Y. The SET2-RPB1 interaction domain of human RECQ5 is important for transcription-associated genome stability. Mol. Cell. Biol., 31, 2090-2099 (2011).

30) Schwendener S, Raynard S, Paliwal S, Cheng A, Kanagaraj R, Shevelev I, Stark JM, Sung P, Janscak P. Physical interaction of RECQ5 helicase with RAD51 facilitates its anti-recombinase activity. J. Biol. Chem., 285, 15739-15745 (2010).

31) Islam MN, Fox D 3rd, Guo R, Enomoto T, Wang W. RecQL5 promotes genome stabilization through two parallel mechanisms-interacting with RNA polymerase II and acting as a helicase. Mol. Cell. Biol., 30, 2460-2472 (2010).

32) Aygün O, Svejstrup J, Liu YA. A RECQ5-RNA polymerase II association identified by targeted proteomic analysis of human chromatin. Proc. Natl. Acad. Sci. U.S.A., 105, 8580-8584 (2008).

33) Kanagaraj R, Saydam N, Garcia PL, Zheng L, Janscak P. Human RECQ5beta helicase promotes strand exchange on synthetic DNA structures resembling a stalled replication fork. Nucleic Acids Res., 34, 5217-5231 (2006).

34) Izumikawa $K$, Yanagida $M$, Hayano $T$, Tachikawa $H$, Komatsu W, Shimamoto A, Futami K, Furuichi Y, Shinkawa T, Yamauchi Y, Isobe T, Takahashi N. Association of human DNA helicase RecQ5beta with RNA polymerase II and its possible role in transcription. Biochem. J., 413, 505-516 (2008).

35) Kanagaraj R, Huehn D, MacKellar A, Menigatti M, Zheng L, Urban V, Shevelev I, Greenleaf AL, Janscak P. RECQ5 helicase associates with the C-terminal repeat domain of RNA polymerase II during productive elongation phase of transcription. Nucleic Acids Res., 38, $8131-8140$ (2010).

36) Zhou G, Liu Y, Wu SY, Tie F, Lou H, Chiang CM, Luo G. Purification of a novel RECQL5-SWI/SNF-RNAPII super complex. Int. J. Biochem. Mol. Biol., 1, 101-111 (2010).

37) Walker JE, Saraste M, Runswick MJ, Gay NJ. Distantly related sequences in the alpha- and beta-subunits of ATP synthase, myosin, kinases and other ATP-requiring enzymes and a common nucleotide binding fold. EMBO J., 1, 945-951 (1982).

38) Islam MN, Paquet N, Fox D 3rd, Dray E, Zheng XF, Klein H, Sung $\mathrm{P}$, Wang W. A variant of the breast cancer type 2 susceptibility protein (BRC) repeat is essential for the RECQL5 helicase to interact with RAD51 recombinase for genome stabilization. J. Biol. Chem., 287, 23808-23818 (2012).

39) Pellegrini L, Yu DS, Lo T, Anand S, Lee M, Blundell TL, Venkitaraman AR. Insights into DNA recombination from the structure of a RAD51-BRCA2 complex. Nature, 420, 287-293 (2002).

40) Carreira A, Kowalczykowski SC. Two classes of BRC repeats in BRCA2 promote RAD51 nucleoprotein filament function by distinct mechanisms. Proc. Natl. Acad. Sci. U.S.A., 108, 10448-10453 (2011). 